\title{
Accuracy of chest computed tomography and reverse transcription polymerase chain reaction in diagnosis of 2019 novel coronavirus disease; a systematic review and meta-analysis
}

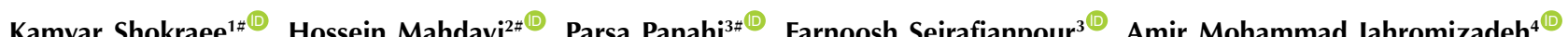

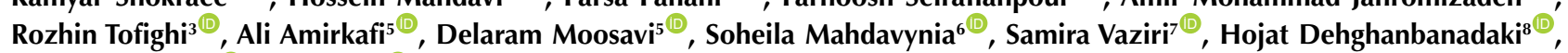
Hossein Aazami $^{\oplus}$, Ali Kabir ${ }^{1 *}$

${ }^{1}$ Minimally Invasive Surgery Research Center, Iran University of Medical Sciences, Tehran, Iran

2Shahid Beheshti University of Medical Sciences, Tehran, Iran

${ }^{3}$ Student Research Committee, Faculty of Medicine, Iran University of Medical Sciences, Tehran, Iran

${ }^{4}$ School of Pharmacy, Tehran University of Medical Sciences, Tehran, Iran

${ }^{5}$ Faculty of Medicine, Iran University of Medical Sciences, Tehran, Iran

${ }^{6}$ Department of Pediatric Nephrology, Firoozabadi Hospital, Iran University of Medical Sciences, Tehran, Iran

'Emergency Medicine Management Research Center, Iran University of Medical Sciences, Tehran, Iran

${ }^{8}$ Metabolic Disorders Research Center, Endocrinology and Metabolism Molecular-Cellular Sciences Institute, Tehran University of Medical Sciences, Tehran, Iran

${ }^{9}$ Endocrinology and Metabolism Research Center, Endocrinology and Metabolism Clinical Sciences Institute, Tehran University of Medical Sciences, Tehran, Iran

"Three authors have contributed equally and all of them are first authors

\section{*Correspondence to}

Ali Kabir, MD, MPH, PhD; Email:

kabir.a@iums.ac.ir, aikabir@

yahoo.com

Received 27 Dec. 2020

Accepted 17 Feb. 2021

Published online 11 Mar. 2021

Keywords: Reverse transcription polymerase chain reaction,

Chest computed tomography, Diagnostic test accuracy metaanalysis, Severe acute respiratory syndrome coronavirus 2 , COVID-19

Citation: Shokraee K, Mahdavi $\mathrm{H}$, Panahi P, Seirafianpour $F$, Jahromizadeh AM, Tofighi R, Amirkafi A, Moosavi D, Mahdavynia S, Vaziri S, Dehghanbanadaki H, Aazami $\mathrm{H}$, Kabir A. Accuracy of chest computed tomography and reverse transcription polymerase chain reaction in diagnosis of 2019 novel coronavirus disease; a systematic review and metaanalysis. Immunopatho Persa. 2021;7(2):e36. DOI:10.34172/ipp.2021.36

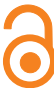

\section{Abstract}

Introduction: This study aims to measure the diagnostic accuracy of chest computed tomography (CT) and reverse transcription polymerase chain reaction assay (RT-PCR) in COVID-19 in a systematic review and metaanalysis.

Methods: PubMed, Scopus, Embase, and Google Scholar, WHO, SSRN, and MedRxiv have been searched on March 26, 2020 for all the alternative names of the disease and virus. Risk of bias assessment was based on QUADAS-2. Data from English-language studies after January 12, 2019 were pooled to calculate necessary diagnostic values and underwent diagnostic test accuracy, random-effects, proportions, and subgroup metaanalysis.

Results: Pooled from 27 included studies, the sensitivity of chest CT was calculated $96.6 \%$, specificity $22.5 \%$, diagnostic odds ratio (DOR) 8.2, positive likelihood ratio (PLR) $1.2(95 \%$ Cl: 1.1-1.4), and negative likelihood ratio (NLR) 0.15 (95\% Cl: $0.1-0.3)$. The sensitivity for initial RT-PCR was $79.7 \%$, the specificity $100 \%$, and NLR 0.18 .

Conclusion: Considering the results, in order to diagnose COVID-19 (coronavirus disease 2019), it is recommended to initially performing chest $\mathrm{CT}$ to rule out the uninfected people. In suspicious cases, we sugges RT-PCR to confirm the disease. Performing serial RT-PCR instead of the one-time test is highly recommended, to let the viral loads reach the diagnostic levels, especially in cases of high clinical suspicion.

\section{Introduction}

Rationale

Coronaviruses are important human pathogens, causing a broad range of conditions from encephalitis to enteritis and more prominent nowadays, pneumonia. The latter seems to be the most frequent and critically severe manifestation of the current severe acute respiratory syndrome coronavirus 2 (SARS-CoV-2), previously known as 2019-nCoV (1,2). This novel coronavirus has infected more people than its two epidemically out-breaking predecessors, SARS-CoV and Middle East respiratory syndrome coronavirus (MERS-CoV), which have had cumulatively 10000 cases so far $(1,3,4)$. The disease occurs mostly in patients $30-79$ years old ( $86.6 \%$ of confirmed cases), and most cases $(81 \%)$ were having a mild set of manifestations (non-pneumonia or mild pneumonia) maximally $(1,5,6)$. Different tests with various accuracies, the results similar to the other infections, and no robust collective diagnostic accuracy all confuse clinicians in

Copyright $(\subset 2021$ The Author(s); Published by Nickan Research Institute. This is an open-access article distributed under the terms of the Creative Commons Attribution License (http://creativecommons.org/licenses/by/4.0), which permits unrestricted use, distribution, and reproduction in any medium, provided the original work is properly cited. 


\section{Key point}

In a systematic review and meta-analysis to measure the diagnostic accuracy of chest computed tomography and reverse transcription polymerase chain reaction assay, we recommended to initially performing chest $\mathrm{CT}$ to rule out the uninfected people. In the suspicious cases, we suggest RT-PCR to confirm the disease. Performing serial reverse transcription polymerase chain reaction instead of the onetime test is highly recommended, to let the viral loads reach the diagnostic levels, especially in cases of high clinical suspicion

the differential approach they must take to cope with these patients. To the best of the authors' knowledge, until the day of this study, there is no systematic analysis to address the diagnostic value of reverse transcription polymerase chain reaction (RT-PCR) and chest computed tomography (CT) tests in 2019 novel coronavirus disease (COVID-19).

\section{Clinical role of the tests, signs, and symptoms}

The predominant symptoms of COVID-19 include fever and cough( 7-9). The symptoms are differentially insignificant, and the most sensitive tools of health care system now makes use of to confirm the infection are chest CT and RT-PCR, respectively $(7,10,11)$. Ground-glass opacity (GGO) and then patchy bilateral shadowing have been the most frequent CT scan findings in the admission process (7). Besides, SARS-CoV-2 manifests some of the same CT characteristics of MERS-CoV and SARS-CoV, such as crazy-paving, pleural effusion, lymphadenopathy, and the absence of pulmonary cavitations (12).

\section{Objectives}

This study aims to use the sets data regarding chest CT and RT-PCR extracted from various studies - executed in China and other involving countries - to achieve a bird'seye view and demonstrate more reliable and robust clinical diagnosis criteria regarding this emergent matter. This is obtained through systematic review and diagnostic test accuracy (DTA) by meta-analysis for CT and RT-PCR. Moreover, the publications in which categorized data of CT findings have been discussed will be described and analyzed.

\section{Methods \\ Protocol and registration}

This study is implemented according to the PRISMA statement (13), its subsequent for DTA meta-analysis (14), and the MOOSE group's proposal for reporting of metaanalysis of observational studies in epidemiology (15).

\section{Eligibility criteria}

The inclusion criteria comprised (a) all population-based studies after December 1, 2019, (b) addressing chest CT and/or RT-PCR tests regarding the SARS-CoV-2 adequately or as a part of their endeavors, (c) with directlyreported or extractable values of sensitivity, specificity, or any statistical/quantitative measurement of the diagnostic quality of the test. The exclusion criteria consisted of (a) all publications not meeting the above, (b) non-English literature, (c) studies before December 1, 2019, (d) case reports, reviews, or descriptive/qualitative studies, and studies in which only a novel diagnostic test has been innovated, and (e) studies in which no mention of any diagnostic tests or a quantitative measurement has been made.

\section{Information sources}

PubMed, Scopus, Embase, and Google Scholar databases have been searched for the evidence. Other sources searched - especially their resources made ready for the current condition - to make use of the additional research were WHO (http://www.who.int/emergencies/diseases/ novel-coronavirus-2019), SSRN (http://papers.ssrn.com), MedRxiv (http://www.medrxiv.org), and CDC (http:// www.cdc.gov/coronavirus/2019-ncov).

\section{Search strategies}

eTable 1 in online Supplementary file 1 shows the search strategies used, designed by AK, FS, HM, KS, and PP, mainly to not limit the entries to any condition, but only to the alternative names that SARS-CoV-2/COVID-19 has been called. AK, HA, and PP started and completed the search on March 26, 2020, and only the articles after December 1, 2019, have been included. Google Scholar, as a cumulative database, was limited only to the first 500 related results.

\section{Data collection process}

Endnote $\mathbb{R}$ X9 (Clarivate Analytics, Philadelphia, USA) was used for study screening and data extraction. AK, FS, HM, KS, and PP assigned each study to the inclusion and exclusion groups. In the first step, each of the five authors has read the titles and abstracts, and if doubted, has evaluated the full-text. Secondly, the five authors read the full-text and executed the final inclusion process. Disagreement situations regarding the inclusion process resolved through dialogue and no necessity for a thirdparty involvement occurred.

\section{Data extraction}

AA, AJ, DM, FS, HA, HD, PP, RT, SM, and SV extracted data, filling a pre-designed spreadsheet containing study characteristics and variables regarding chest CT and RTPCR, subgroups and definitions, categories of chest CT findings, sensitivity, specificity, positive and negative predictive values (PPV and NPV), positive and negative likelihood ratios (PLR and NLR), odds ratio (OR), accuracy, and demographics. Where any of the mentioned values not directly reported in a study, the authors were to calculate it - if possible. 


\section{Quality assessment}

AA, AJ, AK, DM, FS, HA, HD, PP, RT, SM, and SV completed the quality assessment based on the QUADAS-2 revised tool for diagnostic accuracy studies (16). The team reviewed each study and filled the pre-designed table for the risk of bias appraisal and its related concerns in 4 domains: patient selection, index tests (RT-PCR and chest CT), reference standard, and flow and timing. The studies - with acceptable quality - concerning the measurement of RT-PCR and chest CT application for the diagnosis enrolled in the meta-analysis for accuracy of the tests.

\section{Diagnostic accuracy measures}

The accuracy for the diagnosis of COVID-19 per patient has been measured in the studies through true positive (TP), false positive (FP), false negative (FN), and true negative (TN) test measures, which also result in the sensitivity, specificity, PLR, and NLR for RT-PCR and chest CT. The studies have reported the number of participants for the tests with positive/negative results for SARS-CoV-2. They then mostly have compared the test with the gold standard (usually RT-PCR), directly or indirectly. Some of the entries had subgroups defined, of which subgroups of severity were of interest and extracted for further analysis.

\section{Synthesis of results}

The definition for COVID-19 in the included studies mainly comprised symptoms, and the confirmation was mostly by RT-PCR and then chest CT. To build a set of analyzable data, the variables expressing the same concepts in different studies needed to be similarized and interpreted to unified values. The described values regarding chest CT and RT-PCR have been used to fill in or, if need be, calculate TP, FP, FN, TN, sensitivity, specificity, PLR, NLR, PPV, NPV, odds ratio, and their standard errors and 95\% confidence intervals (CIs).

\section{Meta-analysis}

The software used for analyses was Stata/MP version 16.0 (StataCorp LLC, College Station, Texas, USA). AK designed and $\mathrm{AK}, \mathrm{HM}, \mathrm{KS}$, and PP performed the meta-analyses. To be more informative, the data were summarized and pooled in various types. We used the metandi command for pooling the classic studies which have all the necessary information about TP, FP, FN, and TN values for the index tests (17). The second approach for summarizing the existing literature was collecting any index in any evidence and pooling it using metaprop command for numeral variables (18). The third analysis was based on metan command for the indices expressed as means and their standard errors (19).

\section{Other analysis}

The command metabias was used for the appraisal of publication bias and metatrim to trim and fill the studies if needed. For the meta-regression assessment, metareg was executed; metainf was used to investigate the influence of each study on the meta-analysis by omitting the studies one-by-one and repeating the computations. Besides, to appraise the differences of chest CT characteristics based on the severity in two subgroups of non-severe and severe patients, metaprop was performed.

\section{Results}

Study selection

The process of study screening is summarized in the flow diagram (Figure 1). After searching, there were 2074 studies found; (1) PubMed resulted in 428, (2) Scopus in 359, (3) Embase in 757, and (4) Google Scholar in 500 first related, and (5) searching in other sources' data made for the novel coronavirus (WHO, SSRN, MedRxiv, and $\mathrm{CDC}$ ) in 28 entries. After removing the duplicates, 1395 studies enrolled in the title/abstract screening for the eligibility criteria. Ninety-one were accepted for fulltext screening, and then 30 studies were included in the qualitative analysis (The excluded entries were; two basic sciences and molecular assessment, two case reports, one epidemiologic study, two letters to the editor, one nonaccessible full-text, eight non-English, eight reviews, 33 not mentioning any quantitative measures of the index tests, and four according to other reasons). Of 30 selected studies, all were addressing the diagnostic values of chest CT and two, RT-PCR.

\section{Study characteristics}

eTable 2 in the online supplement summarizes the study characteristics. Of 30 included studies, all were conducted in Asia; 29 (96.7\%) in China and 1 (3.3\%) in South Korea. The set contained the following study designs: 13 (43.3\%) retrospective cross-sectional (20-32), 7 (23.3\%) retrospective cohort $(10,11,33-37), 4(13.3 \%)$ prospective cross-sectional (1,38-40), 3 (10.0\%) prospective cohort (41-43), and three (10.0\%) retrospective case series $(9,44,45)$. No randomized controlled trial was eligible to include. The reference standards in the studies were the following; metagenomic next-generation sequencing (mNGS) in 2 studies (6.7\%), RT-PCR in 22 (73.3\%), chest CT in 1 (3.3\%), and clinical features in three (10.0\%). Participants mostly recruited from hospitals.

\section{Risk of bias and applicability}

Figure 2 shows the QUADAS-2 risk of bias and concerns summary, and eFigure 1 in the online supplement presents the graph. The studies mostly had a high/unclear risk of bias in all four domains. In the course of patient selection, the studies mostly included the infected population and did not randomize or blind the inclusion process. Besides, non-optimal reference standards - more often RT-PCR, which is itself not an ideal standard - have been creating a source of bias. Consequently, FP and TP values are not completely reliable, and thus the accuracy values would not be so valid (most probably overestimated). A major 


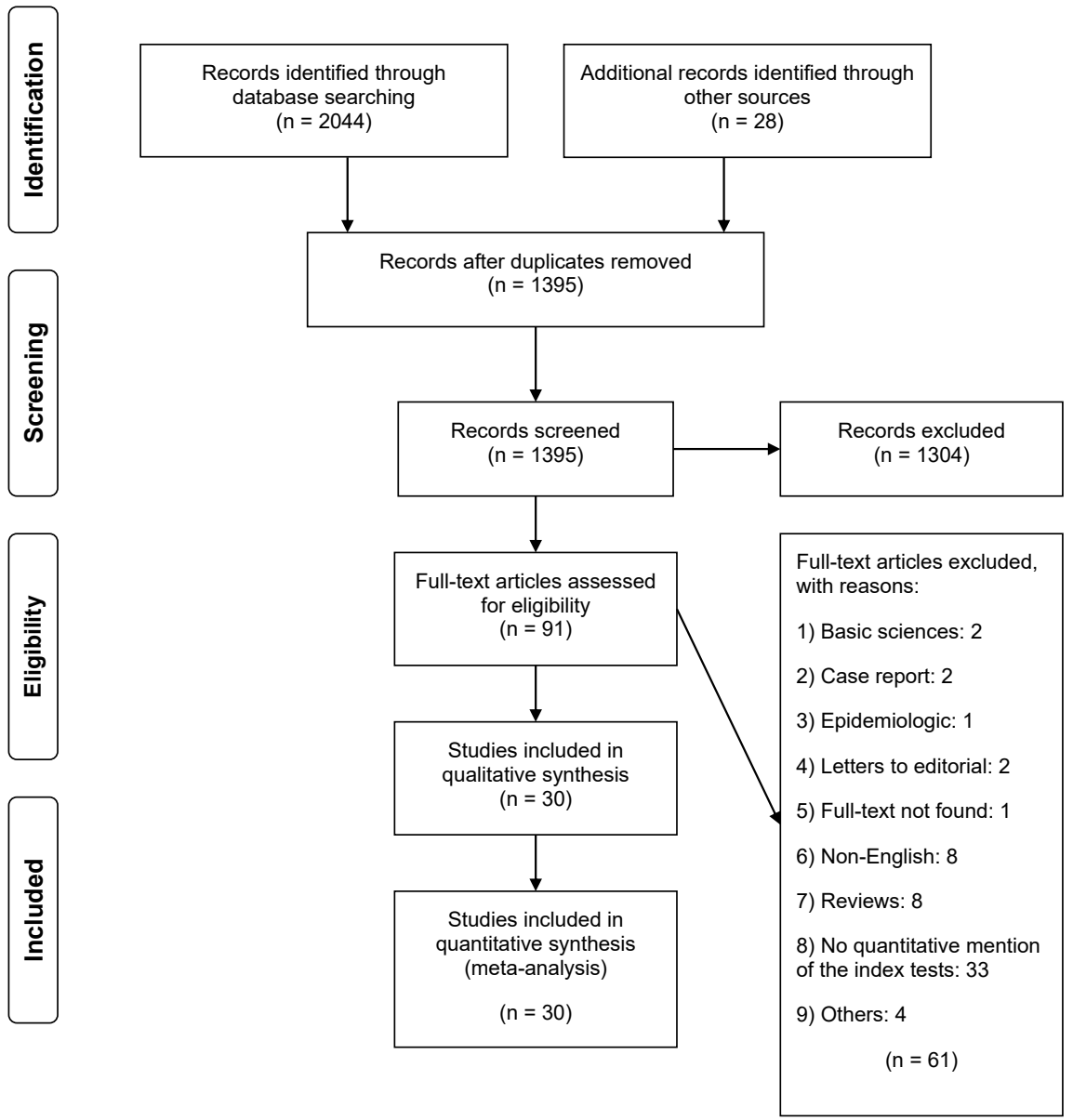

Figure 1. The Flow Diagram of the study inclusion process. From Moher et al (13).

source of bias in flow and timing domain would be raised from the time interval between the reference standard and the index test, which has jeopardized the validity of the comparison between the reference and index tests because of the disease progression and therapies during the time. Also, the studies mostly were implemented in clinical settings; therefore the interpretation of the index test would have been exposed to bias since the reference test results had probably been revealed to the clinician. Conclusively, the overall risk of bias for most of the studies would be high or unclear, affecting the diagnostic values.

\section{Results of individual studies}

Four studies were reporting adequate values to be included in DTA analysis for chest CT. Ai et al (41) calculated a sensitivity of $100 \%$ (95\% CI: 83.9 to 100$)$, Tao Ai et al (10) $96.5 \%$ (95\% CI: 94.7 to 97.7 ), Liu et al (46) $96.7 \%$ (95\% CI: 94.7 to 97.9), and Yang et al (30) 100\% (95\% CI: 64.6 to 100) for chest CT (eTable 3). Another set of four studies were eligible for DTA for initial RT-PCR, with the sensitivity in the study of Ai et al (41) of $66.7 \%$ (95\% CI: 48.1 to 85.5 ), Ai et al (10) $88.3 \%$ (95\% CI: 74.3 to 89.6), Bernheim et al (20) 88.2 (95\% CI: 80.5 to 93.1), and Fang et al (11) $70.6 \%$ (95\% CI: 57.0 to 81.3) (eTable 4).

\section{Synthesis of results}

Chest CT

Studies addressing sensitivity, specificity, NLR, PLR, NPV, PPV, accuracy, and OR of chest CT directly or indirectly were extracted and used for fixed- or random-effects meta-analysis - which one of them fitted best. The results are shown in eTable 5 in the online supplement, and the Forest plot for the sensitivity could be found in Figure 3 .

The four studies $(10,26,37,41)$ of interest for DTA metaanalysis contained extracted values of TP, FP, FN, and TN were pooled and underwent metandi. The results are shown in the online supplement's eTable 6 . The sensitivity was $96.6 \%$ (95\% CI: 95.1 to 97.6$)$, specificity $22.5 \%$ (95\% CI: 1.4 to 34.5 ), and NLR 0.15 (95\% CI: 0.1 to 0.3 ). Figure 4 presents the hierarchical summary receiver operating characteristic (HSROC) curve for the DTA analysis, which demonstrates that the study estimates of the aggregated sensitivity and specificity (alternatively, the accuracy) are heterogeneous.

The proportions meta-analysis of chest CT characteristics indicated that 97\% (95\% CI: 95 to 99) of COVID-19 patients had abnormalities on their chest CT. These abnormalities were bilateral in 82\% (95\% CI: 76 to 87 ) and involved more than two lobes in $74 \%$ (95\% CI: 


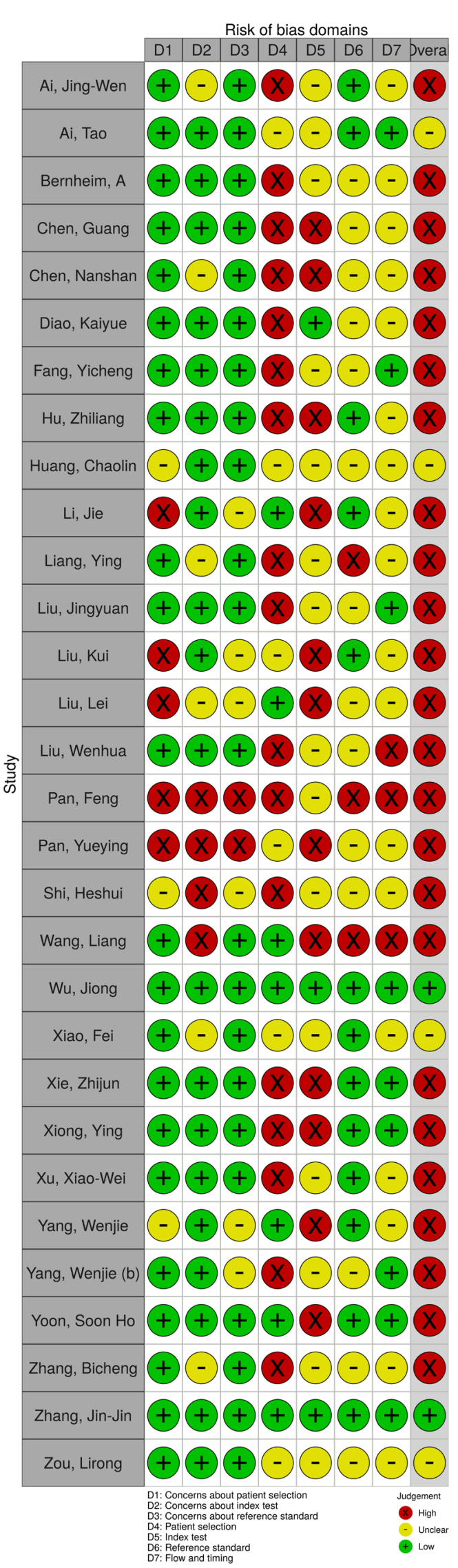

Figure 2. Risk of bias and applicability concerns summary; Created by robvis; Luke A McGuinness (2019). robvis: An R package and web application for visualising risk-of-bias assessments. https://github.com/mcguinlu/robvis
53 to 96). The most affected lobe of the lungs was right lower lobe in 54\% (95\% CI: 2 to 100), and the most frequent pattern was mixed GGO, which is seen in $71 \%$ (95\% CI: 36 to 92) of patients. Other CT characteristics are summarized in the online supplement's eTable 7 . In additional analyses, after merging the patterns that are equivalent to GGO or consolidation, results demonstrated that GGO or consolidation are seen in 65\% (95\% CI: 49 to 80 ). Additionally, random-effects meta-analysis after merging similar characteristics in chest CT showed that the peripheral distribution, linear opacities, and pleural effusion are seen in 35\% (95\% CI: 16 to 54), 49\% (95\% CI: 8 to 89 ) and $8 \%$ (95\% CI: 5 to 11$)$ of patients, respectively (Table 1).

The CT scan characteristics were not different between severe and non-severe patients except for the number of pleural effusion findings (eTable 8); 0.86 for the severe patients (95\% CI: 0.46 to 0.97 ) and 0.02 for the non-severe group (95\% CI: 0.00 to 0.12 ).

\section{RT-PCR}

Pooling diagnostic values of two studies $(23,41)$ for RTPCR by metan regardless of being serial or only as an initial test show sensitivity and specificity of $88.2 \%$ (95\% CI - 78.1 to 98.2 ) and $100.0 \%$ (95\% CI: 97.6 to 102.4 ), respectively (eTable 9). I-square indicated no heterogeneity between these two studies.

To assess the initial RT-PCR, four studies $(10,11,20,41)$ have been pooled by metan and the sensitivity and specificity were $79.7 \%$ (95\% CI: 70.8 to 88.5 ) and $100 \%$ (95\% CI: 97.0 to 103.0 ), respectively, with the I-square of $68.8 \%$ showing heterogeneous data for the sensitivity $(P=0.022)$, but not for the other values (eTable 9).

Diagnostic values of two studies $(11,41)$ have been pooled by metan for second-time RT-PCR. The sensitivity of $67.6 \%$ (95\% CI: 38.7 to 96.6 ) and accuracy of $89.2 \%$ (95\% CI: 78.7 to 99.7$)$ obtained, with I-square demonstrating heterogeneous data for sensitivity (eTable 9).

\section{Additional analyses \\ Chest CT}

Publication bias for the studies addressing chest CT showed bias for the sensitivity and PPV $(P=0.002$ and 0.044 , respectively). No trimming was performed in both cases, because of no significant changes in data. The metainf analysis showed no deviations from 95\% confidence ranges for the diagnostic values of chest CT. Meta-regression for the diagnostic values of CT showed that of all independent variables, the mean age of the study samples $\left(P=0.098\right.$; $\left.\operatorname{tau}^{2}=57.53\right)$ and percentage of males $\left(P=0.14\right.$; $\left.\operatorname{tau}^{2}=56.99\right)$ had significant effects on the heterogeneity of sensitivity between the studies. This effect vanished in the combination of mean age and percentage of males $\left(P=0.33\right.$ and 0.40 , respectively; tau $\left.^{2}=61.82\right)$. Thus, the mean age of samples and sex combinations of the population have partially affected the heterogeneity. 


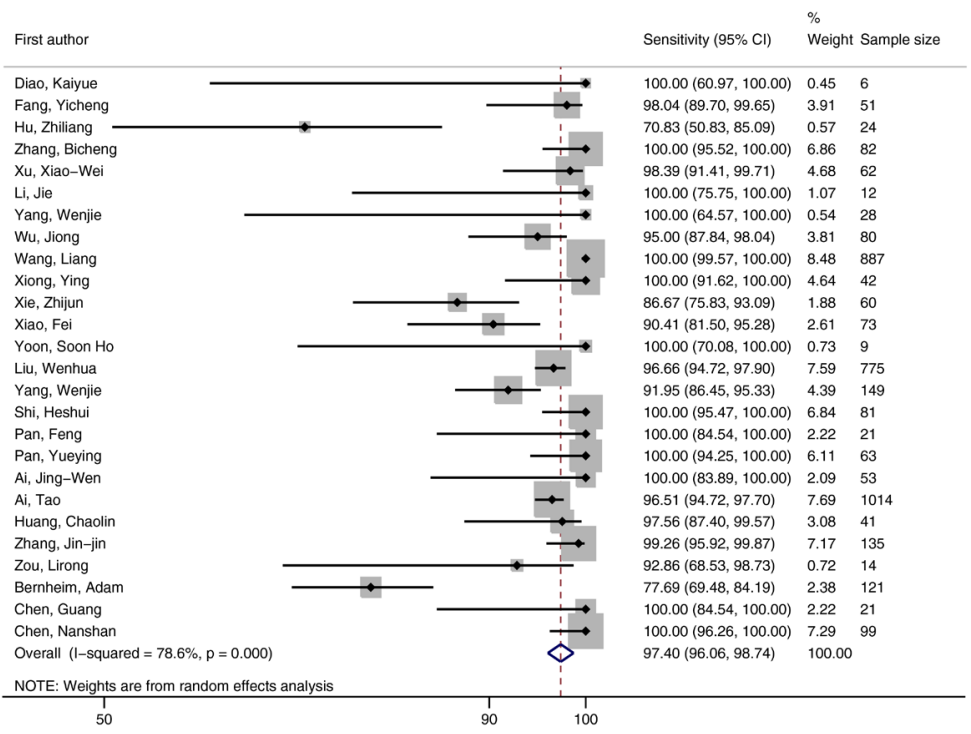

Figure 3. Forest plot for the sensitivity of the chest CT studies.

The results showed no significant effect of the sample size, reference standard, study design, and ethnicity, as independent variables, alone or in combinations, on the diagnostic values.

\section{RT-PCR}

The assessment for publication bias, trim and fill, and meta-regression for the pooled data of RT-PCR were not applicable since the number of studies was insufficient. For initial RT-PCR, studies underwent metabias and had significant publication bias for sensitivity $(P<0.001)$ and accuracy $(P=0.002)$. After metatrim, no trimming was performed, and data were unchanged. Command metainf showed no deviations from the $95 \%$ confidence range.

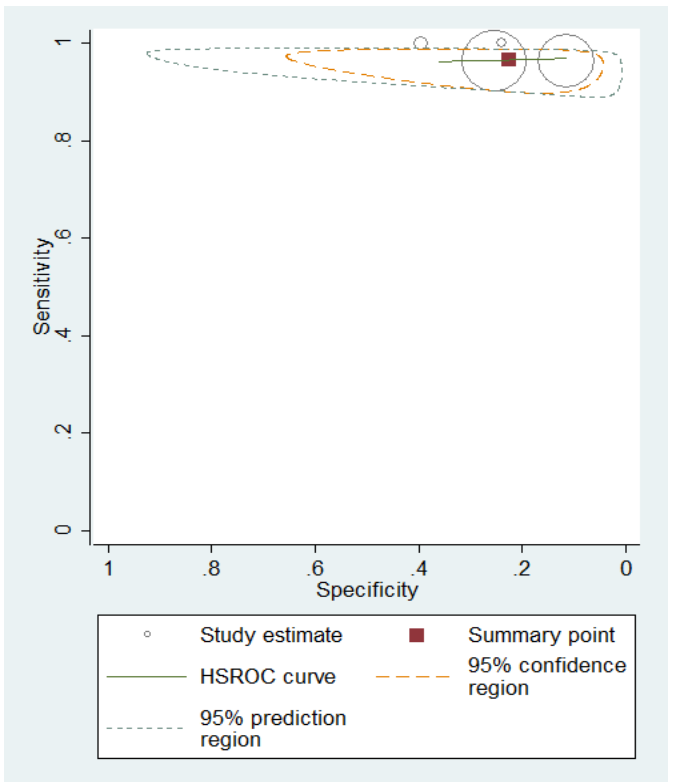

Figure 4. Hierarchical summary receiver operating characteristic (HSROC) curve for the random-effects meta-analysis of chest CT.

\section{Discussion}

\section{Chest CT}

The results of data analysis in this study indicate that the CT scan has high sensitivity but relatively low-specificity, because of a low false-negative rate and a high falsepositive rate. Therefore, many non-infected people are misdiagnosed, while on the other hand, not many of the patients with COVID-19 are missed. Besides, CT is an acceptable test for ruling out COVID-19, while not a robust tool for confirming the disease. As mentioned before, the false-negative rate was low in CT because the virus involved the lung in the early stages of the disease $(20,35,36)$. Furthermore, a high false-positive rate in CT may be because of the similar behavior of SARSCoV-2 to the other respiratory tract viruses (influenza, parainfluenza, and respiratory syncytial viruses) $(10,26,37)$. GGO, pleural effusion, consolidations, treein-bud patterns, and nodules are common findings in all (47). The high DOR and sensitivity, relatively high NPV and accuracy, in combination with low NLR for chest CT show that it has a high diagnostic value. However, we should consider that all diagnostic indices are somehow affected by bias in the domains of reference standard, index test, and flow and timing.

The analysis demonstrates that GGO is one of the most

Table 1. Results of the proportions meta-analysis for the aggregate data of chest CT characteristics

\begin{tabular}{lccc}
\hline Characteristics & $\begin{array}{c}\text { \% Findings } \\
(\mathbf{9 5} \% \mathbf{~ C l})\end{array}$ & $\begin{array}{c}\text { No. of } \\
\text { studies }\end{array}$ & $\begin{array}{c}\text { Total sample } \\
\text { size }\end{array}$ \\
\hline $\begin{array}{l}\text { Ground-glass opacity or } \\
\text { consolidation }\end{array}$ & $65(49,80)$ & 12 & 1102 \\
Peripheral distribution & $35(16,54)$ & 7 & 720 \\
Linear opacity & $49(8,89)$ & 3 & 37 \\
Pleural effusion & $8(5,11)$ & 4 & 322 \\
\hline
\end{tabular}


frequent patterns in COVID-19 patients. Still, it is very unspecific, with differential diagnoses such as cancer, inflammatory conditions, injuries, edema, hemorrhage, and pulmonary fibrosis( 48). More importantly, GGO could be found as a widespread finding in $\mathrm{H} 1 \mathrm{~N} 1$ influenza patients (49). It also has been found in MERS-CoV and SARS-CoV patients and all types of viral lower respiratory infections, more frequently in the infection caused by parainfluenza and respiratory syncytial viruses (47,50-52). Mixed GGO was also evident in $71 \%$ of CT findings, which is also could be found frequently in SARS and malignancies $(51,53,54)$. In the context of the involvement's symmetry and loci, chest tomography findings were more frequently bilateral. Lower lobes of both right and left sides were the most involved lobes, which is a similar trait to that of the parainfluenza virus but separates COVID-19 form influenza and respiratory syncytial infections (47). The distribution is mostly peripheral. Results of the subgroup analysis have shown a higher prevalence of pleural effusion in the severe than the non-severe COVID-19 patients. However, pleural effusion in COVID-19 is majorly overlapped with its frequent differential diagnoses; congestive heart failure, parapneumonic effusion, malignancy, pulmonary embolization, and other viral diseases [55]. Besides, bilateral pleural effusion is a common finding in patients with viral lower respiratory tract infection caused by MERS-CoV, parainfluenza virus, respiratory syncytial virus, and influenza virus $(47,50)$. Thus, this finding may be of high value for severity monitoring and follow-up. To the day, there have been not many studies addressing CT characteristics in COVID-19, and more subgroup analyses are needed to bring more findings similar to the pleural effusion to light, making a valuable asset of diagnostic evidence.

\section{Diagnostic role of RT-PCR and chest CT}

According to the RT-PCR test indices, it seems a better tool than CT for confirming the disease, but because of the large number of undiagnosed patients in this procedure, it cannot be used suitably for ruling out the disease. Thus, RT-PCR is not a suitable tool for the primary screening of patients. The higher false-negative ratio in RT-PCR is due to sampling errors, sampling location, and low viral load of the sample (10). However, these results are not reliable due to few numbers of studies that entered in the analysis and their result was completely different. Yang et al (37) showed that PCR sensitivity increased in the serial RTPCR due to an increase in viral load in the sample from the patients. Still, Ai et al (41) demonstrated that the sensitivity of second-time PCR is lower than the initial PCR. It seems the first study designed a better method, and its results are more reliable. Therefore second-time RT-PCR may be a suitable tool to follow up the patients whose test results are negative but are clinically suspicious. Given all the aspects, further studies are needed to conclude the application of second-time RT-PCR. On the other hand, there were no available data about the time interval between the symptoms' onset and initial RT-PCR, or initial and second RT-PCR. The mean age of the samples has affected the heterogeneity among the studies, and thus it is essential to interpret and design the diagnostic studies based on the age groups, especially the elderly. Besides, as a source of heterogeneity of the studies, the quality assessment in all four domains is not promising; it has a massive effect on CT and RT-PCR's diagnostic values and causes overestimation of the CT sensitivity. The time interval between RT-PCR and CT, and patient selection methods are the most significant concerns. Henceforward, this study could not suggest a definite time for performing RT-PCR and an optimal interval between RT-PCR and chest CT, and further evaluation is needed to suggest the best time to perform the tests.

\section{Limitations and strengths}

The novel nature and the pandemic situation of COVID-19 urges us to rapidly address a diagnostic review to facilitate the clinical approaches, though not enough time seems to be passed from the first cases, allowing more in-depth evaluations and more populated closed-cases resourcing the studies. This has limited us not only because of the number and quality of the studies reviewed but also in terms of not fully comprehending the real behavior of the virus.

It is essential to mention that along with the quantitative data in the studies, we even used the descriptive data regarding RT-PCR and CT, compiling them quantitatively, to extract data needed to calculate our index values. Another strength of our work is having different studies from different locations. This enabled us to compute PPV and NPV. Usually, these indices are not calculated in diagnostic test accuracy meta-analysis because of the different values. However, we calculated these indices by using metan in addition to metandi even though interpreting these two indices should be conducted very cautiously because of the high impact of the prevalence in each region on such indices.

Notably, the prevalence of the disease has a high impact on PPV and NPV, and the aggregate data for RT-PCR have not led to PLR and NLR. Moreover, the prevalence could result in different PPV and NPV in different locations, because of the wide variety of numbers of closed cases and different stages of the involvement of every location. Thus, time is needed to attain more closed cases and more detailed studies of RT-PCR and chest CT.

\section{Conclusion}

The pandemic situation and high rates of disease transmission urge us to rapidly diagnose the patients and isolate them to stop further spreading. The results show that with its very good NLR, sensitivity, availability, and rapidness, chest $\mathrm{CT}$ scan is an excellent test to rule out COVID-19 in the uninfected. It also is a very good 
follow-up and severity assessment tool. Our analysis confirms this through the high NPV of chest CT. Besides, noting that RT-PCR is a specific but not adequately rapid and available test, it could be used to confirm the suspicious cases after performing the initial chest CT scan. However, considering the high rates of false-negative in the initial RT-PCR tests, in case of strong suspicion, it is recommended to perform repeated RT-PCR tests, to have an absolute confirmation by letting the viral load reach higher levels. Performing repeated RT-PCR is crucial in these situations to avoid missing the infected people, and to stop the further spread of the disease.

\section{Authors' contribution}

AK was the principal investigator of the study. AK, FS, HM, KS and PP were included in preparing the concept and design. AK, $\mathrm{HM}, \mathrm{KS}$ and PP rechecked the manuscript and critically evaluated the intellectual contents. All authors participated in preparing the final draft of the manuscript, revised the manuscript and critically evaluated the intellectual contents. All authors have read and approved the content of the manuscript and confirmed the accuracy or integrity of any part of the work.

\section{Conflicts of interest}

The authors declare that they have no competing interests.

\section{Ethical considerations}

The protocol has been approved by the Iran University of Medical Sciences' Research and Ethics Committee (\#IR.IUMS. REC.1399.055). Ethical issues (including plagiarism, data fabrication, double publication) have been completely observed by the authors.

\section{Funding/Support}

None.

Supplementary Materials

Online Supplementary file 1 contains eFigure 1 and eTables 1-9.

\section{References}

1. Huang C, Wang Y, Li X, Ren L, Zhao J, Hu Y, et al. Clinical features of patients infected with 2019 novel coronavirus in Wuhan, China. Lancet. 2020;395:497-506. doi: 10.1016/ S0140-6736(20)30183-5.

2. Chang D, Lin M, Wei L, Xie L, Zhu G, Dela Cruz CS, et al. Epidemiologic and Clinical Characteristics of Novel Coronavirus Infections Involving 13 Patients Outside Wuhan, China. JAMA. 2020;323:1092-1093. doi: 10.1001/ jama.2020.1623.

3. World Health Organization. Coronavirus disease 2019 (COVID-19) Situation Report - 98 2020;27 April.

4. Mahase E. Coronavirus covid-19 has killed more people than SARS and MERS combined, despite lower case fatality rate. BMJ. 2020;368:m641. doi: 10.1136/bmj.m641.

5. Epidemiology Working Group for NCIP Epidemic Response, Chinese Center for Disease Control and Prevention. [The epidemiological characteristics of an outbreak of 2019 novel coronavirus diseases (COVID-19) in China]. Zhonghua Liu Xing Bing Xue Za Zhi. 2020;41(2):145-151. Chinese. doi: 10.3760/cma.j.issn.0254-6450.2020.02.003.

6. Wu Z, McGoogan JM. Characteristics of and Important Lessons From the Coronavirus Disease 2019 (COVID-19) Outbreak in China: Summary of a Report of 72314 Cases From the Chinese Center for Disease Control and Prevention.
JAMA. 2020;323:1239-42. doi: 10.1001/jama.2020.2648.

7. Guan WJ, Ni ZY, Hu Y, Liang WH, Ou CQ, He JX, et al; China Medical Treatment Expert Group for Covid-19. Clinical Characteristics of Coronavirus Disease 2019 in China. N Engl J Med. 2020;382:1708-20. doi: 10.1056/NEJMoa2002032.

8. Lai CC, Shih TP, Ko WC, Tang HJ, Hsueh PR. Severe acute respiratory syndrome coronavirus 2 (SARS-CoV-2) and coronavirus disease-2019 (COVID-19): The epidemic and the challenges. Int J Antimicrob Agents. 2020;55:105924. doi: 10.1016/j.ijantimicag.2020.105924.

9. $\mathrm{Xu} X \mathrm{~W}, \mathrm{Wu} X \mathrm{X}$, Jiang $\mathrm{XG}, \mathrm{Xu} \mathrm{KJ}$, Ying $\mathrm{LJ}, \mathrm{Ma} C \mathrm{CL}$, et al. Clinical findings in a group of patients infected with the 2019 novel coronavirus (SARS-Cov-2) outside of Wuhan, China: retrospective case series. BMJ. 2020;368:m606. doi: 10.1136/ bmj.m606.

10. Ai T, Yang Z, Hou H, Zhan C, Chen C, Lv W, et al. Correlation of Chest CT and RT-PCR Testing for Coronavirus Disease 2019 (COVID-19) in China: A Report of 1014 Cases. Radiology. 2020;296:E32-E40. doi: 10.1148/radiol.2020200642.

11. Fang $\mathrm{Y}$, Zhang $\mathrm{H}, \mathrm{Xie} J$, Lin $\mathrm{M}$, Ying $\mathrm{L}$, Pang $\mathrm{P}$, et al. Sensitivity of Chest CT for COVID-19: Comparison to RT-PCR. Radiology. 2020;296:E115-7. doi: 10.1148/radiol.2020200432.

12. Chung $M$, Bernheim A, Mei X, Zhang N, Huang M, Zeng $X$, et al. CT Imaging Features of 2019 Novel Coronavirus (2019-nCoV). Radiology. 2020;295(:202-7. doi: 10.1148/ radiol.2020200230

13. Moher D, Liberati A, Tetzlaff J, Altman DG; PRISMA Group. Preferred reporting items for systematic reviews and meta-analyses: the PRISMA statement. PLoS Med. 2009;6(7):e1000097. doi: 10.1371/journal.pmed.1000097.

14. Mclnnes MDF, Moher D, Thombs BD, McGrath TA, Bossuyt PM, Group and the P-D. Preferred Reporting Items for a Systematic Review and Meta-analysis of Diagnostic Test Accuracy Studies: The PRISMA-DTA Statement. JAMA. 2018;319:388-96. doi: 10.1001/jama.2017.19163.

15. Stroup DF, Berlin JA, Morton SC, Olkin I, Williamson GD, Rennie D, et al. Meta-analysis of observational studies in epidemiology: a proposal for reporting. Meta-analysis Of Observational Studies in Epidemiology (MOOSE) group. JAMA. 2000;283:2008-12. doi: 10.1001/jama.283.15.2008.

16. Whiting PF, Rutjes AWS, Westwood ME, Mallett S, Deeks JJ, Reitsma JB, et al. QUADAS-2: a revised tool for the quality assessment of diagnostic accuracy studies. Ann Intern Med. 2011;155:529-36. doi: 10.7326/0003-4819-155-8201110180-00009.

17. Harbord RM, Whiting P. Metandi: Meta-analysis of diagnostic accuracy using hierarchical logistic regression. Stata J. 2009;9:211-29. doi: 10.1177/1536867x0900900203.

18. Nyaga VN, Arbyn M, Aerts M. Metaprop: A Stata command to perform meta-analysis of binomial data. Arch Public Health. 2014:72:1-10. doi: 10.1186/2049-3258-72-39.

19. Harris RJ, Deeks JJ, Altman DG, Bradburn MJ. Metan: fixedand random-effects meta-analysis. Stata J. 2008:3-28.

20. Bernheim A, Mei X, Huang M, Yang $Y$, Fayad ZA, Zhang $\mathrm{N}$, et al. Chest CT Findings in Coronavirus Disease-19 (COVID-19): Relationship to Duration of Infection. Radiology. 2020;295:200463. doi: 10.1148/radiol.2020200463.

21. Chen G, Wu D, Guo W, Cao Y, Huang D, Wang $H$, et al. Clinical and immunological features of severe and moderate coronavirus disease 2019. J Clin Invest. 2020;130(5):26202629. doi: $10.1172 / \mathrm{JCl} 137244$.

22. Hu Z, Song C, Xu C, Jin G, Chen $Y, X u X$, et al. Clinical characteristics of 24 asymptomatic infections with COVID-19 screened among close contacts in Nanjing, China. Sci China Life Sci. 2020;63:706-711. doi: 10.1007/s11427-020-1661-4

23. Liang Y, Liang J, Zhou Q, Li X, Lin F, Deng Z, et al. Prevalence and clinical features of 2019 novel coronavirus disease 
(COVID-19) in the Fever Clinic of a teaching hospital in Beijing: a single-center, retrospective study. MedRxiv. 2020. doi: 10.1101/2020.02.25.20027763.

24. Liu K, Fang YY, Deng Y, Liu W, Wang MF, Ma JP, et al. Clinical characteristics of novel coronavirus cases in tertiary hospitals in Hubei Province. Chin Med J (Engl). 2020;133(9):1025-31. doi: 10.1097/CM9.0000000000000744.

25. Wan S, Xiang Y, Fang W, Zheng Y, Li B, Hu Y, et al. Clinical features and treatment of COVID-19 patients in northeast Chongqing. J Med Virol. 2020;92:797-806. doi: 10.1002/ jmv. 25783

26. Wei Y, Lu Y, Xia L, Yuan X, Li G, Li X, et al. Analysis of 2019 novel coronavirus infection and clinical characteristics of outpatients: An epidemiological study from a fever clinic in Wuhan, China. J Med Virol. 2020;92:2758-67. doi: 10.1002/ jmv.26175.

27. Wu J, Wu X, Zeng W, Guo D, Fang Z, Chen L, et al. Chest CT Findings in Patients With Coronavirus Disease 2019 and Its Relationship With Clinical Features. Invest Radiol. 2020;55:257-261. doi: 10.1097/RLI.0000000000000670.

28. Xie Z, Bao J, Cai Z, Liu S, Chen H, Qi J, et al. Clinical characteristics of 60 COVID-19-infected patients with or without renal injury in Hangzhou, China. SSRN ELibrary 2020.

29. Xiong Y, Sun D, Liu Y, Fan Y, Zhao L, Li X, et al. Clinical and High-Resolution CT Features of the COVID-19 Infection: Comparison of the Initial and Follow-up Changes. Invest Radiol. 2020;55:332-339. doi: 10.1097/RLI.0000000000000674.

30. Yang W, Cao Q, Qin L, Wang X, Cheng Z, Pan A, et al. Clinical characteristics and imaging manifestations of the 2019 novel coronavirus disease (COVID-19):A multi-center study in Wenzhou city, Zhejiang, China. J Infect. 2020;80:388-393. doi: 10.1016/j.jinf.2020.02.016.

31. Zhang B, Zhou X, Qiu Y, Feng F, Feng J, Jia Y, et al. Clinical characteristics of 82 death cases with COVID-19. MedRxiv 2020. doi: 10.1101/2020.02.26.20028191.

32. Zhang JJ, Dong $X$, Cao $Y Y$, Yuan YD, Yang YB, Yan YQ, et al. Clinical characteristics of 140 patients infected with SARSCoV-2 in Wuhan, China. Allergy. 2020;75(7):1730-41. doi: 10.1111/all.14238.

33. Chen $N$, Zhou M, Dong $X, Q u$ J, Gong $F$, Han $Y$, et al. Epidemiological and clinical characteristics of 99 cases of 2019 novel coronavirus pneumonia in Wuhan, China: a descriptive study. Lancet. 2020;395:507-513. doi: 10.1016/ S0140-6736(20)30211-7.

34. Li J, Li S, Cai Y, Liu Q, Li X, Zeng Z, et al. Epidemiological and Clinical Characteristics of 17 Hospitalized Patients with 2019 Novel Coronavirus Infections Outside Wuhan, China. MedRxiv. 2020. doi: 10.1101/2020.02.11.20022053.

35. Pan F, Ye T, Sun P, Gui S, Liang B, Li L, et al. Time Course of Lung Changes at Chest CT during Recovery from Coronavirus Disease 2019 (COVID-19). Radiology. 2020;295:715-721. doi: 10.1148/radiol.2020200370.

36. Shi H, Han X, Jiang N, Cao Y, Alwalid O, Gu J, et al. Radiological findings from 81 patients with COVID-19 pneumonia in Wuhan, China: a descriptive study. Lancet Infect Dis. 2020;20:425-434. doi: 10.1016/S1473-3099(20)300864.

37. Yang W, Cheng Z, Cao Q, Yang Z, Zhou H, Qin L, et al. Clinical Features and Computed Tomographic Manifestations of Patients with 2019 Novel Coronavirus Pneumonia: A Single Center Study in Shanghai, China. SSRN ELibrary; 2020.

38. Pan Y, Guan H, Zhou S, Wang Y, Li Q, Zhu T, et al. Initial $\mathrm{CT}$ findings and temporal changes in patients with the novel coronavirus pneumonia (2019-nCoV): a study of 63 patients in Wuhan, China. Eur Radiol. 2020;30:3306-3309. doi: 10.1007/ s00330-020-06731-x.
39. Xiao $F$, Tang $M$, Zheng $X$, Liu $Y$, Li $X$, Shan $H$. Evidence for Gastrointestinal Infection of SARS-CoV-2. Gastroenterology. 2020:158(6):1831-1833.e3. doi: 10.1053/j. gastro.2020.02.055

40. Zou L, Ruan F, Huang M, Liang L, Huang H, Hong Z, et al. SARS-CoV-2 Viral Load in Upper Respiratory Specimens of Infected Patients. N Engl J Med. 2020;382:1177-9. doi: 10.1056/NEJMc2001737.

41. Ai JW, Zhang HCHY, Xu T, Wu J, Zhu M, Yu YQ, et al. Optimizing diagnostic strategy for novel coronavirus pneumonia, a multi-center study in Eastern China. MedRxiv 2020. doi: 10.1101/2020.02.13.20022673.

42. Liu J, Liu Y, Xiang P, Pu L, Xiong H, Li C, et al. Neutrophilto-lymphocyte ratio predicts critical illness patients with 2019 coronavirus disease in the early stage. J Transl Med. 2020;18:206. doi: 10.1186/s12967-020-02374-0.

43. Wang L, Li Q. Clinical and imaging evidence of Wuhan-viral pneumonia. A large-scale prospective cohort study. SSRN ELibrary; 2020.

44. Diao K, Han P, Pang T, Li Y, Yang Z. HRCT imaging features in representative imported cases of 2019 novel coronavirus pneumonia. Precis Clin Med. 2020;3:9-13 doi: 10.1093/ pcmedi/pbaa004.

45. Yoon SH, Lee KH, Kim JY, Lee YK, Ko H, Kim KH, et al. Chest Radiographic and CT Findings of the 2019 Novel Coronavirus Disease (COVID-19): Analysis of Nine Patients Treated in Korea. Korean J Radiol. 2020;21:494-500. doi: 10.3348/ kjr.2020.0132.

46. Liu K, Fang YY, Deng Y, Liu W, Wang MF, Ma JP, et al. Clinical characteristics of novel coronavirus cases in tertiary hospitals in Hubei Province. Chin Med J (Engl). 2020;133:1025-1031. doi: 10.1097/CM9.0000000000000744.

47. Kim MC, Kim MY, Lee HI, Lee SO, Choi SH, Kim YS, et al. CT findings in viral lower respiratory tract infections caused by parainfluenza virus, influenza virus and respiratory syncytial virus. Medicine (Baltimore). 2016;95:e4003. doi: 10.1097/ MD.0000000000004003.

48. Yu H, Liu S, Zhang C, Li S, Ren J, Zhang J, et al. Computed tomography and pathology evaluation of lung ground-glass opacity. Exp Ther Med. 2018;16:5305-9. doi: 10.3892/ etm.2018.6886.

49. Schoen K, Horvat N, Guerreiro NFC, de Castro I, de Giassi KS Spectrum of clinical and radiographic findings in patients with diagnosis of $\mathrm{H} 1 \mathrm{~N} 1$ and correlation with clinical severity. BMC Infect Dis. 2019;19:964. doi: 10.1186/s12879-019-4592-0.

50. Ajlan AM, Ahyad RA, Jamjoom LG, Alharthy A, Madani TA. Middle East respiratory syndrome coronavirus (MERS$\mathrm{CoV}$ ) infection: chest CT findings. AJR Am J Roentgenol. 2014;203:782-7. doi: 10.2214/AJR.14.13021.

51. Wang R, Sun H, Song L, Song W, Cui H, Li B, et al. [Plain radiograph and $C T$ features of 112 patients with SARS in acute stage]. Beijing Da Xue Xue Bao Yi Xue Ban. 2003;35:29-33.

52. Jin Z, You $\mathrm{H}$, Zhang $\mathrm{W}$, Wang $\mathrm{Y}$, Liang J, Mu W, et al. [Thoracic high resolution CT findings of 100 SARS patients in convalescent period]. Zhongguo Yi Xue Ke Xue Yuan Xue Bao 2003;25:512-5.

53. Infante M, Lutman RF, Imparato S, Di Rocco M, Ceresoli GL, Torri V, et al. Differential diagnosis and management of focal ground-glass opacities. Eur Respir J. 2009;33:821-7. doi: 10.1183/09031936.00047908.

54. Lei L, Jian-ya G, Hu W, Zhang X, Gua L, Liu C, et al. Clinical characteristics of 51 patients discharged from hospital with COVID-19 in Chongqing, China. MedRxiv 2020. doi: 10.1101/2020.02.20.20025536

55. Light RW. Pleural Effusions. Med Clin North Am 2011;95:105570. doi: doi: 10.1016/j.mcna.2011.08.005. 